\title{
Observational Study of Prescribing Pattern of Antiepileptic Drugs in Tertiary Care Hospital
}

\author{
M. Deepalakshmi, Megha Gupta, Sunil Ahuja \\ JSS College of Pharmacy, Udhagamandalam, Tamil Nadu
}

\begin{abstract}
Trial Design: Observational study

Methods

Inclusion criteria: All In-patients who were treated with AEDs admitted in different units of Medicine Department.

Exclusion Criteria: All pregnant women who are on AEDs.

Sample size: 35

Objective: The chief objective behind this project was to observe the various therapy of AED used in epilepsy and in some co-morbid condition and to observe the patient condition.

Outcome

- A total of 35 patients data were recorded in the study for 1 month.

- In the study percentage of men suffering from epilepsy was $45.71 \%$ (16) and Female was $54.24 \%(19)$.

- The study shows that most of the patients 14 (40\%) were from the age group of 31-60 years; followed by $14(40 \%)$ of patients in the age group of 61-90 years and 7 (20\%) patients were in the group of 10-30 year.

- Most commonly prescribed drug concluded is phenytoin which accounts for 30 (85.30\%) in both the genders, followed by phenobarbitone 11 (31.18\%) and levitracetam usage was 11 (31.18\%)

- $\quad$ Monotherapy is the type of therapy most frequently used in 23 patients $(65.71 \%)$.

- $\quad$ Dual therapy is used in 6 patient (17.14\%).Triple therapy is used in 5 patient (14.28\%).

Conclusion: The most frequently prescribed AED in the study was Phenytoin followed by Phenobarbital and levitracetam.
\end{abstract}

\section{Introduction}

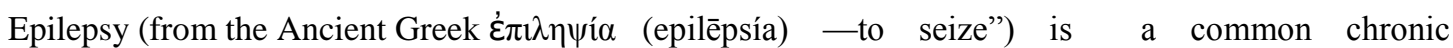
neurological disorder characterized by recurrent unprovoked seizures. Epilepsy is believed to affect $1 \%$ to $2 \%$ of the population; approximately 2 million people have been diagnosed with epilepsy. The incidence is highest in childhood and old age. The prognosis is good if the patient adheres strictly to prescribed treatment.

Antiepileptic drug therapy is the mainstay of treatment for most patients with epilepsy. Seizure classification is an important element in designing the treatment plan, since some antiepileptic drugs have different activities against various seizure types. The International League Against Epilepsy classify the three main types of seizures: partial, generalized, and unclassified.

Amongst the various factors affecting anti-epileptic drug (AED) usage, the major determinants are type of epilepsy, age and gender of patient, side effect profile and availability of medicines, affordability of the patient, and preference of the treating physician as well as the practice setting. Attempt to control epilepsy is done using mono and poly therapy. Due to the long duration of treatment, various adverse reactions (ADRs) are seen, which require change of medication and monitoring. A recent study in Bangalore, India, reported that the problem is nearly two and half times higher in rural areas as compared to urban areas, where they are not receiving any treatment.

Begin with monotherapy about $50 \%$ to $70 \%$ of patients can be maintained on one antiepileptic drug (AED), but all are not seizure free.Up to $60 \%$ of patients with epilepsy are noncompliant, and this is the most common reason for treatment failure.Drug therapy may not be indicated in patients who have had only one seizure or those whose seizures have minimal impact on their lives. Patients who have had two or more seizures should generally be started on AEDs.

Factors favoring successful withdrawal of AEDs include a seizure-free period of 2 to 4 years, complete seizure control within 1 year of onset, an onset of seizures after age 2 years and before age 35 years, and a normal EEG.

Many controlled clinical trials have tested the efficacy of the older Anti-Epileptic Drugs (AEDs), (such as Phenobarbital and Phenytoin) and newer AEDs (such as Carbamazepine and Valproic acid) in controlling seizure frequency and their safety when prescribed in monotherapy or in combination. 
The interest in drug utilization studies began in the early 1960s, and its importance has increased since then because of increase in marketing of new drugs, wide variation in the pattern of drug prescribing and consumption, growing concern about delayed adverse effects and the increasing concern regarding the cost of drugs.

Purpose of the study is to investigate the use of antiepileptic drugs in epilepsy, Changes in prescription patterns, exposure of specific drugs to certain patient groups, combination of drugs,

generic substitution, and potential for drug abuse and quantify the use of antiepileptic drugs (AEDs) in epilepsy and other disorders to be investigated in a tertiary care hospital

\section{Background}

An epileptic seizure is an episode of neurologic dysfunction in which abnormal neuronal firing is manifest clinically by changes in motor control, sensory perception, behavior, and/or autonomic function. Epilepsy is the condition of recurrent spontaneous seizures arising from aberrant electrical activity within the brain. While anyone can experience a seizure under the appropriate pathophysiological conditions, epilepsy suggests an enduring alteration of brain function that facilitates abnormal neuronal firing.

The aberrant electrical activity that underlies epilepsy is the result of biochemical processes at the cellular level promoting neuronal hyperexcitability and neuronal hypersynchrony. However, a single neuron, discharging abnormally, is insufficient to produce a clinical seizure, which occurs only in the context of large neuronal networks. Cortical and several key subcortical structures are involved in generating a seizure

Antiepileptic drug therapy is the mainstay of treatment for most patients with epilepsy. Seizure classification is an important element in designing the treatment plan, since some antiepileptic drugs have different activities against various seizure types. The International League Against Epilepsy classify the three main types of seizures: partial, generalized, and unclassified.

Epilepsy is believed to affect $1 \%$ to $2 \%$ of the population; approximately 2 million people have been diagnosed with epilepsy. The incidence is highest in childhood and old age. The prognosis is good if the patient adheres strictly to prescribed treatment.

\section{Causes}

- About half of all epilepsy cases are idiopathic; possible causes of other cases include:

- Birth trauma (inadequate oxygen supply to the brain, blood incompatibility, or hemorrhage)

- Perinatal infection

- Anoxia

- Infectious diseases (meningitis, encephalitis, or brain abscess)

- Ingestion of toxins (mercury, lead, or carbon monoxide)

- Brain tumors

- Inherited disorders or degenerative disease, such as phenylketonuria or tuberous sclerosis

- Head injury or trauma

- Metabolic disorders, such as hypoglycemia and hypoparathyroidism

- Cerebrovascular accident (hemorrhage, thrombosis, or embolism).

\section{Classification of seizure types}

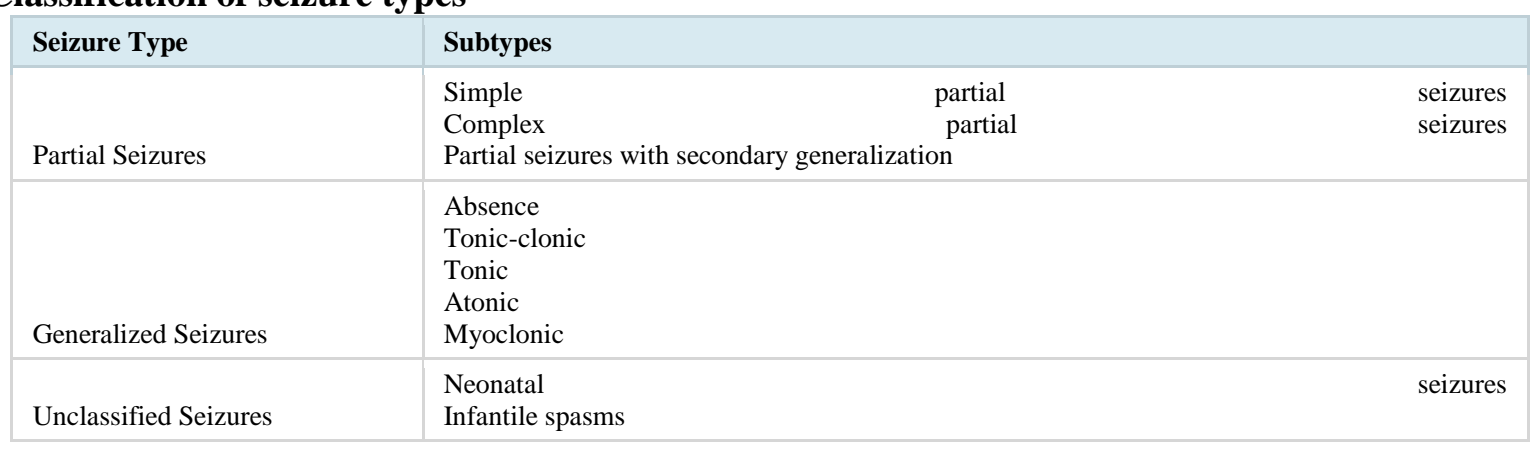

\section{Pathophysiology}

Some neurons in the brain may depolarize easily or be hyperexcitable; this epileptogenic focus fires more readily thannormal when stimulated. In these neurons, the membrane potential at rest is less negative or inhibitory connections are missing, possibly as a result of decreased gamma-amino butyric acid (GABA) activity or localized shifts in electrolytes. 
On stimulation, the epileptogenic focus fires and spreads electrical current to surrounding cells. These cells fire in turn and the impulse cascades to one side of the brain (a partial seizure), both sides of the brain (a generalized seizure), or cortical, subcortical, and brain stem areas.

The brain's metabolic demand for oxygen increases dramatically during a seizure. If this demand isn't met, hypoxia and brain damage ensue. Firing of inhibitory neurons causes the excitatory neurons to slow their firing and eventually stop. If this inhibitory action doesn't occur, the result is status epilepticus: one seizure occurring right after another and another; without treatment the anoxia is fatal.

\section{Signs and symptoms}

The hallmark of epilepsy is recurring seizures, which can be classified as partial, generalized, status epilepticus, or unclassified (some patients may be affected by more than one type).

\section{Complications}

Complications may include:

- Hypoxia or anoxia from airway occlusion

- Traumatic injury

- Brain damage

- Depression and anxiety.

\section{Diagnosis}

Clinically, the diagnosis of epilepsy is based on the occurrence of one or more seizures, and proof or the assumption that the condition that caused them is still present. Diagnostic tests that help support the findings include:

Computed tomography or magnetic resonance imaging reveal abnormalities.

Electroencephalogram (EEG) reveals paroxysmal abnormalities to confirm the diagnosis and provide evidence of the continuing tendency to have seizures. In tonic-clonic seizures, high, fast voltage spikes are present in all leads; in absence seizures, rounded spike wave complexes are diagnostic. A negative EEG doesn't rule out epilepsy,because the abnormalities occur intermittently.

Skull X-ray may show evidence of fractures or shifting of the pineal gland, bony erosion, or separated sutures. Serum chemistry blood studies may reveal hypoglycemia, electrolyte imbalances, elevated liver enzymes, and elevated alcohol levels, providing clues to underlying conditions that increase the risk of seizure activity.

\section{Treatment}

Treatment may include: drug therapy specific to the type of seizure, including phenytoin, carbamazepine, phenobarbital, and primidone for generalized tonic-clonic seizures and complex partial seizures - I.V. fosphenytoin (Cerebyx) is an alternative to phenytoin (Dilantin) that is just as effective, with a long halflife and minimal CNS depression (stable for 120 days at room temperature and compatible with many frequently used I.V. solutions; can be administered rapidly without the adverse cardiovascular effects that occur with phenytoin) valproic acid, clonazepam, and ethosuximide for absence seizures gabapentin (Neurontin) and felbamate as other anticonvulsant drugs surgical removal of a demonstrated focal lesion, if drug therapy is ineffective surgery to remove the underlying cause, such as a tumor, abscess, or vascular problem vagus nerve stimulator implant may help reduce the incidence of focal seizure I.V. diazepam, lorazepam, phenytoin, or phenobarbital for status epilepticus administration of dextrose (when seizures are secondary to hypoglycemia) or thiamine (in chronic alcoholism or withdrawal).

\section{Important characteristics of antiepileptic medications}

\begin{tabular}{|c|c|c|c|c|c|}
\hline Drug Name & $\begin{array}{l}\text { Mechanism of } \\
\text { Action }\end{array}$ & $\begin{array}{l}\text { Seizure } \\
\text { Types } \\
\text { Treated }\end{array}$ & Adverse Effects & Drug Interactions & $\begin{array}{l}\text { Generic } \\
\text { Available }\end{array}$ \\
\hline Carbamazepine & $\begin{array}{l}\mathrm{Na}+\quad \text { channel } \\
\text { inhibition }\end{array}$ & $\begin{array}{l}\text { Partial } \\
\text { Tonic- } \\
\text { Clonic }\end{array}$ & $\begin{array}{l}\text { Neurological: dizziness, } \begin{array}{r}\text { diplopia, } \\
\text { ataxia, }\end{array} \\
\text { Non-Neurological: aplastic anemia, } \\
\text { leucopenia, gastrointestinal irritation, } \\
\text { hepatotoxicity, hyponatremia, skin } \\
\text { rash* }\end{array}$ & $\begin{array}{l}\text { Enzyme Substrate: } \\
\text { CYP 3A4, 2C8 } \\
\text { Enzyme Inducer: } \\
\text { CYP 1A2, 2B6, } \\
\text { 2C8, 2C9, 2C19, } \\
\text { 3A4 } \\
\text { Enzyme Inhibitor: } \\
\text { None }\end{array}$ & Yes \\
\hline Clonazepam & $\begin{array}{l}\text { Potentiate GABA } \\
\text { receptor function }\end{array}$ & $\begin{array}{l}\text { Absence } \\
\text { Atypical } \\
\text { Absence }\end{array}$ & $\begin{array}{l}\text { Neurological: ataxia, sedation, lethargy } \\
\text { Non-Neurological: anorexia }\end{array}$ & $\begin{array}{lr}\text { Enzyme } & \text { Substrate: } \\
\text { CYP } & 3 \mathrm{~A} 4 \\
\text { Enzyme } & \text { inducer: }\end{array}$ & No \\
\hline
\end{tabular}




\begin{tabular}{|c|c|c|c|c|c|}
\hline & & Myoclonic & & $\begin{array}{l}\text { None } \\
\text { Enzyme Inhibitor: } \\
\text { None }\end{array}$ & \\
\hline Ethosuximide & $\begin{array}{l}\text { T-type } \mathrm{Ca} 2+ \\
\text { channel inhibition } \\
\text { in thalamus }\end{array}$ & Absence & $\begin{array}{l}\text { Neurological: ataxia, lethargy, } \\
\text { headache } \\
\text { Non-Neurological: gastrointestinal } \\
\text { irritation, skin rash, bone marrow } \\
\text { suppression }\end{array}$ & $\begin{array}{lr}\text { Enzyme } & \text { Substrate: } \\
\text { CYP } & 3 \mathrm{~A} 4 \\
\text { Enzyme } & \text { inducer: } \\
\text { None } & \\
\text { Enzyme } & \text { Inhibitor: } \\
\text { None } & \end{array}$ & $\begin{array}{l}\text { Yes, Only } \\
\text { Available in } \\
\text { Generic }\end{array}$ \\
\hline Felbamate & $\begin{array}{lr}\text { NMDA receptor } \\
\text { antagonist } & \text { and } \\
\text { increase } & \text { GABA } \\
\text { availability } & \end{array}$ & $\begin{array}{l}\text { Partial } \\
\text { Lennox- } \\
\text { Gastaut }\end{array}$ & $\begin{array}{l}\text { Neurological: insomnia, dizziness, } \\
\text { sedation, } \\
\text { Non-Neurological: aplastic anemia, } \\
\text { hepatic failure, weight loss, } \\
\text { gastrointestinal irritation }\end{array}$ & $\begin{array}{l}\text { Enzyme Substrate: } \\
\text { CYP 2E1, 3A4 } \\
\text { Enzyme inducer: } \\
\text { CYP } \\
\text { Enzyme Inhibitor: } \\
\text { CYP 2C19 }\end{array}$ & $\begin{array}{l}\text { No, but } \\
\text { Patent } \\
\text { Expired } \\
9 / 26 / 09\end{array}$ \\
\hline Gabapentin & $\begin{array}{l}\text { GABA analogue } \\
\text { for alpha-2 delta } \\
\text { subunit }\end{array}$ & Partial & 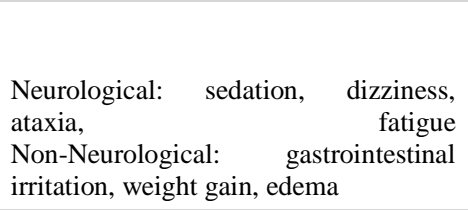 & $\begin{array}{ll}\text { Enzyme } & \text { Substrate: } \\
\text { None } & \\
\text { Enzyme } & \text { Inducer: } \\
\text { None } & \\
\text { Enzyme } & \text { Inhibitor: } \\
\text { None } & \end{array}$ & Yes \\
\hline Lacosamide & $\begin{array}{l}\mathrm{Na}+\quad \text { channel } \\
\text { inhibition }\end{array}$ & Partial & $\begin{array}{l}\text { Neurological: headache, dizziness, } \\
\text { diplopia, ataxia, fatigue, tremor, } \\
\text { somnolence, blurred rision } \\
\text { Non-Neurological: Nausea, vomiting, } \\
\text { diarrhea }\end{array}$ & $\begin{array}{lr}\text { Enzyme } & \text { Substrate: } \\
\text { CYP } & \text { 2C19 } \\
\text { Enzyme } & \text { inducer: } \\
\text { None } & \\
\text { Enzyme Inhibitor: } \\
\text { CYP 2C19 }\end{array}$ & No \\
\hline Lamotrigine & $\begin{array}{l}\text { Decrease } \\
\text { glutamate release }\end{array}$ & $\begin{array}{l}\text { Partial } \\
\text { Tonic- } \\
\text { Clonic } \\
\text { Atypical } \\
\text { Absence } \\
\text { Myoclonic } \\
\text { Lennox- } \\
\text { Gastaut }\end{array}$ & $\begin{array}{l}\text { Neurological: dizziness, diplopia, } \\
\text { sedation, ataxia, headache } \\
\text { Non-Neurological: skin rash* }\end{array}$ & $\begin{array}{l}\text { Enzyme Substrate: } \\
\text { UGT1A4 } \\
\text { Enzyme inducer: } \\
\text { None } \\
\text { Enzyme Inhibitor: } \\
\text { None }\end{array}$ & Yes \\
\hline Levetiracetam & $\begin{array}{l}\text { Synaptic vesicle } \\
\text { release } \\
\text { modulation }\end{array}$ & Partial & $\begin{array}{l}\text { Neurological: sedation, } \begin{array}{r}\text { fatigue, } \\
\text { psychosis }\end{array} \\
\text { incoordination, } \\
\text { Non-Neurological: anemia, leucopenia }\end{array}$ & $\begin{array}{l}\text { Enzyme Substrate: } \\
\text { None } \\
\text { Enzyme inducer: } \\
\text { None } \\
\text { Enzyme Inhibitor: } \\
\text { None }\end{array}$ & Yes \\
\hline Oxcarbazepine & $\begin{array}{l}\mathrm{Na}+\quad \text { channel } \\
\text { inhibition }\end{array}$ & Partial & $\begin{array}{l}\text { Neurological: fatigue, ataxia, dizziness, } \\
\text { diplopia } \\
\text { Non-Neurological: aplastic anemia, } \\
\text { leucopenia, gastrointestinal irritation, } \\
\text { hepatotoxicity, hyponatremia, skin rash }\end{array}$ & $\begin{array}{lr}\text { Enzyme } & \text { Substrate: } \\
\text { CYP } & \\
\text { Enzyme } & \text { Inducer: } \\
\text { CYP } & 3 \mathrm{~A} 4 \\
\text { Enzyme } & \text { Inhibitor: } \\
\text { CYP 2C19 }\end{array}$ & Yes \\
\hline Phenobarbital & $\begin{array}{l}\text { Potentiate GABA } \\
\text { receptor function }\end{array}$ & $\begin{array}{l}\text { Partial } \\
\text { Tonic- } \\
\text { Clonic }\end{array}$ & $\begin{array}{l}\text { Neurological: sedation, } \begin{array}{l}\text { ataxia, } \\
\text { confusion, dizziness, decreased libido, } \\
\text { depression } \\
\begin{array}{l}\text { Non-Neurological: } \\
\text { hepatotoxicity }\end{array}\end{array} \text { Skin rash, }\end{array}$ & $\begin{array}{l}\text { Enzyme Substrate: } \\
\text { CYP 2C9, 2C19, } \\
2 \mathrm{E} 1 \\
\text { Enzyme Inducer: } \\
\mathrm{CYP} 1 \mathrm{~A} 2, \quad 2 \mathrm{~A} 6, \\
\text { 2B6, 2C8, 2C9, 3A4 } \\
\text { Enzyme Inhibitor: } \\
\text { None }\end{array}$ & $\begin{array}{l}\text { Yes, Only } \\
\text { Available in } \\
\text { Generic }\end{array}$ \\
\hline Phenytoin & $\begin{array}{l}\mathrm{Na}+\text { and } \mathrm{Ca} 2+ \\
\text { channel inhibition }\end{array}$ & $\begin{array}{l}\text { Partial } \\
\text { Tonic- } \\
\text { Clonic }\end{array}$ & $\begin{array}{l}\begin{array}{l}\text { Neurological: dizziness, } \\
\text { ataxia, }\end{array} \\
\begin{array}{r}\text { diplopia, } \\
\text { confusion } \\
\text { gon-Neurological: }\end{array} \\
\text { hyperplasia, peripheral } \\
\begin{array}{l}\text { ginmal } \\
\text { neuropathy, } \\
\text { hirsutism, }\end{array} \\
\begin{array}{l}\text { osteomalacia, hepatotoxicity, facial } \\
\text { coarsening, skin rash* }\end{array}\end{array}$ & $\begin{array}{l}\text { Enzyme Substrate: } \\
\text { CYP 2C9, 2C19, } \\
\text { 3A4 } \\
\text { Enzyme Inducer: } \\
\text { CYP 2B6, 2C8, } \\
\text { 2C9, 2C19, 3A4 } \\
\text { and } \\
\text { Enzyme Inhibitor: } \\
\text { None }\end{array}$ & Yes \\
\hline Pregabalin & $\begin{array}{l}\text { GABA analogue } \\
\text { for alpha-2 delta } \\
\text { subunit }\end{array}$ & Partial & $\begin{array}{l}\text { Neurological: ataxia, somnolence, } \\
\text { dizziness, blurred vision, diplopia } \\
\text { Non-Neurological: peripheral edema, } \\
\text { increased appetite }\end{array}$ & $\begin{array}{l}\text { Enzyme Substrate: } \\
\text { None } \\
\text { Enzyme Inducer: } \\
\text { None }\end{array}$ & No \\
\hline
\end{tabular}




\begin{tabular}{|c|c|c|c|c|c|}
\hline & & & & $\begin{array}{l}\text { Enzyme Inhibitor: } \\
\text { None }\end{array}$ & \\
\hline Primidone & $\begin{array}{l}\text { Inhibition of } \\
\text { neuronal firing }\end{array}$ & $\begin{array}{l}\text { Partial } \\
\text { Tonic- } \\
\text { Clonic }\end{array}$ & $\begin{array}{l}\text { Neurological: sedation, ataxia, } \\
\text { confusion, dizziness, decreased libido, } \\
\text { depression } \\
\text { Non-Neurological: Skin rash }\end{array}$ & $\begin{array}{l}\text { Enzyme Substrate: } \\
\text { None } \\
\text { Enzyme inducer: } \\
\text { CYP 1A2, 2B6, } \\
2 \mathrm{C} 8,2 \mathrm{C} 9, \quad 3 \mathrm{~A} 4 \\
\text { Enzyme Inhibitor: } \\
\text { None }\end{array}$ & Yes \\
\hline Rufinamide & $\begin{array}{l}\mathrm{Na}+\quad \text { channel } \\
\text { inhibition }\end{array}$ & $\begin{array}{l}\text { Lennox- } \\
\text { Gastaut }\end{array}$ & $\begin{array}{l}\text { Neurological: headache, dizziness, } \\
\text { fatigue, somnolence, convulsion, } \\
\text { diplopia, tremor, nystagmus } \\
\text { Non-Neurological: nausea, vomiting, } \\
\text { nasopharyngitis, blurred vision }\end{array}$ & $\begin{array}{lr}\text { Enzyme } & \text { Substrate: } \\
\text { CYP } & 3 \mathrm{~A} 4 \\
\text { Enzyme } & \text { inducer: } \\
\text { None } & \\
\text { Enzyme } & \text { Inhibitor: } \\
\text { None } & \end{array}$ & No \\
\hline Tiagabine & $\begin{array}{l}\text { Increase GABA } \\
\text { availability }\end{array}$ & $\begin{array}{l}\text { Partial } \\
\text { Tonic- } \\
\text { Clonic }\end{array}$ & 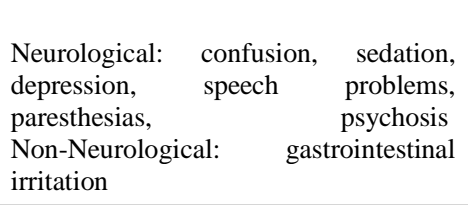 & $\begin{array}{lr}\text { Enzyme } & \text { Substrate: } \\
\text { CYP } & 3 \mathrm{~A} 4 \\
\text { Enzyme } & \text { inducer: } \\
\text { None } & \\
\text { Enzyme } & \text { Inhibitor: } \\
\text { None } & \end{array}$ & No \\
\hline Topiramate & $\begin{array}{l}\mathrm{Na}+\quad \text { channel } \\
\text { inhibition }\end{array}$ & $\begin{array}{l}\text { Partial } \\
\text { Tonic- } \\
\text { Clonic } \\
\text { Lennox- } \\
\text { Gastaut }\end{array}$ & $\begin{array}{l}\text { Neurological: psychomotor slowing, } \\
\text { sedation, speech problems, fatigue, } \\
\text { paresthesias } \\
\text { Non-Neurological: kidney stones, } \\
\text { glaucoma, weight loss, hypohydrosis }\end{array}$ & $\begin{array}{lr}\text { Enzyme } & \text { Substrate: } \\
\text { None } & \\
\text { Enzyme } & \text { inducer: } \\
\text { CYP } & 3 \mathrm{~A} 4 \\
\text { Enzyme Inhibitor: } \\
\text { CYP 2C19 }\end{array}$ & Yes \\
\hline Valproic Acid & $\begin{array}{lr}\text { T-type } & \mathrm{Ca}++ \\
\text { channel } & \text { inhibition } \\
\text { in } & \text { thalamus } \\
\text { increase } & \text { GABA } \\
\text { availability }\end{array}$ & $\begin{array}{l}\text { Partial } \\
\text { Tonic- } \\
\text { Clonic } \\
\text { Absence } \\
\text { Atypical } \\
\text { Absence } \\
\text { Myoclonic }\end{array}$ & $\begin{array}{l}\text { Neurological: ataxia, sedation, tremor } \\
\text { Non-Neurological: Hepatotoxicity, } \\
\text { thrombocytopenia, gastrointestinal } \\
\text { irritation, weight } \\
\text { hyperammonemia }\end{array}$ & $\begin{array}{l}\text { Enzyme Substrate: } \\
\text { UGT 1A6, 1A9, } \\
\text { 2B7, beta-oxidation } \\
\text { Enzyme Inducer: } \\
\text { CYP r 2A6 } \\
\text { Enzyme Inhibitor: } \\
\text { CYP 2C9, 2C19, } \\
\text { 2D6, 3A4 }\end{array}$ & Yes \\
\hline Vigabatrin & $\begin{array}{l}\text { Analog of GABA, } \\
\text { inhibits GABA } \\
\text { catabolism }\end{array}$ & $\begin{array}{l}\text { Complex } \\
\text { Partial }\end{array}$ & $\begin{array}{l}\text { Neurological: headache, fatigue, } \\
\text { drowsiness, dizziness, tremor, } \\
\text { agitation, visual field defects, abnormal } \\
\text { vision, } \\
\text { Non-Neurological: nausea, vomiting, } \\
\text { diarrhea, weight gain, skin rash }\end{array}$ & $\begin{array}{l}\text { Enzyme Substrate: } \\
\text { None } \\
\text { Enzyme inducer: } \\
\text { None } \\
\text { Enzyme Inhibitor: } \\
\text { None }\end{array}$ & No \\
\hline Zonisamide & $\begin{array}{l}\mathrm{Na}+\quad \text { channel } \\
\text { inhibition }\end{array}$ & Partial & $\begin{array}{l}\text { Neurological: sedation, dizziness, } \\
\text { confusion, headache, psychosis } \\
\text { Non-Neurological: Anorexia, renal } \\
\text { stones, hypohydrosis }\end{array}$ & $\begin{array}{l}\text { Enzyme Substrate: } \\
\text { CYP 2C19, 3A4 } \\
\text { Enzyme Inducer: } \\
\text { None } \\
\text { Enzyme Inhibitor: } \\
\text { None }\end{array}$ & Yes \\
\hline
\end{tabular}

Legend: $\mathrm{Ca} 2+=$ calcium ion, $\mathrm{CYP}=$ cytochrome $\mathrm{P}$ enzyme, GABA = gamma amino butyric acid, $\mathrm{Na}+=$ sodium ion, NMDA= N-methyl D-aspartic acid. * denotes skin rash risk (Steven's Johnson syndrome and Toxic Epidermal Necrolysis) related to Human Leukocyte Antigen (HLA)-phenotype.

Over the last decade newer antiepileptic drugs that have been approved for use by the Food and Drug Administration (FDA) in the treatment of epilepsy. While most newer antiepileptic drugs are approved as second line agents for the treatment of refractory seizures, topiramate, oxcarbazepine and lamotrigine are also approved for monotherapy in certain situations.

\section{Methodology}

Study Site: This study is being conducted at Fortis Escorts hospital, Amritsar. It is a Tertiary Care Superspeciality Hospital.

Study Design: This was a hospital based observational study conducted on in-patients to review the current prescribing pattern of antiepileptic drugs in patients with epilepsy admitted to medicine wards.

Sample Size: A total of 35 In-patients from different units of medicine department, who were on AEDs and fulfilled the inclusion criteria were selected and the rest of the patients were excluded from the study, and the data were collected in a well designed proforma. 
Study period: The observational study was conducted for one month.

\section{Study Criteria:}

- Inclusion Criteria: All In-patients who were treated with AEDs admitted in different units of Medicine Department.

- Exclusion Criteria: All pregnant women who are on AEDs.

Source of Data: Data was collected using a well-designed patient data collection form.

- By reviewing the patient's treatment chart, case sheets of the patients.

- From the Medical Record Department.

Preparation of data collection form: Information extracted from the case files will include: Demographic data ,If he/she is a known case of epilepsy and diagnosis (provisional or confirmatory). Treatment: AEDs prescribed and prescription of the AEDs by generic names. The recommended dosages of the AEDs were obtained from the patient case files and discharge summary Drugs which reduce seizure threshold.

Statistical method.

The data of each case file was collected and analyzed by a Suitable method.

\section{Gender}

\section{Results \& Interpretation}

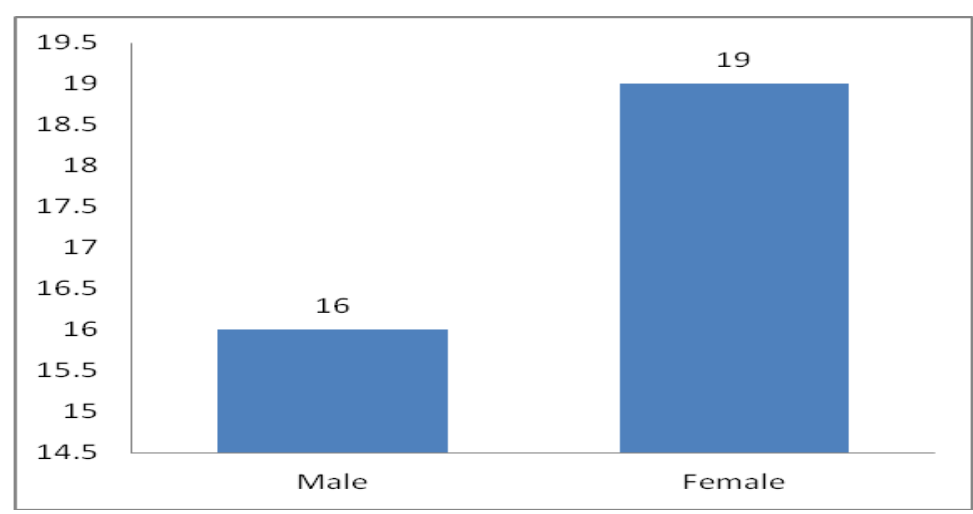

\section{Interpretation}

After study on the epileptic patients, following data were collected:

- Total no. patients: 35

- Total no. of male: 16

- Total no. of female:19

Age

\section{Interpretation}

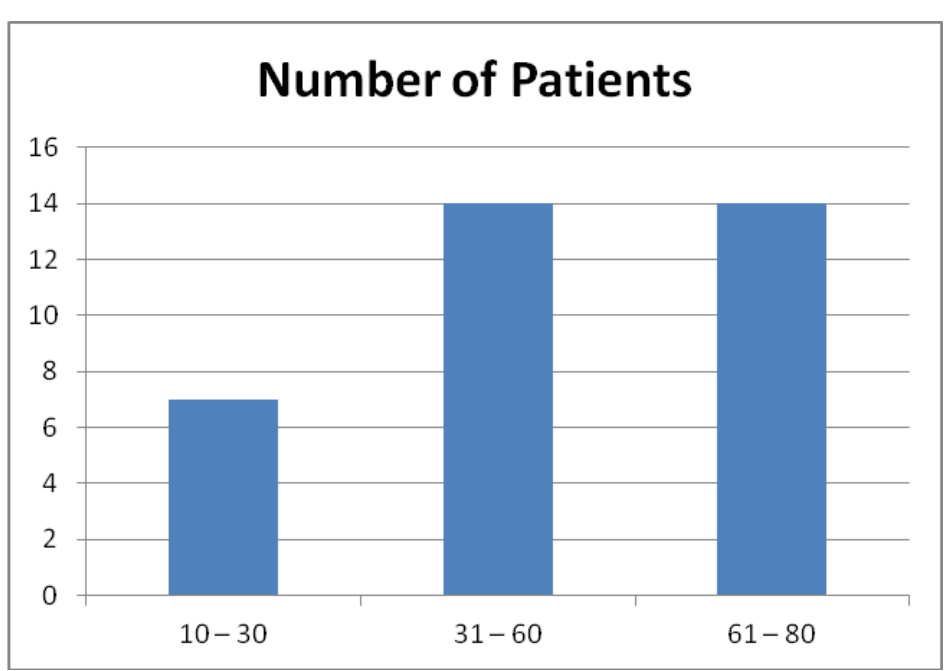

- Total no of patients that come under the age group 10-30 is 7

- Total no of patients that come under the age group 31-60 is 14

- Total no of patients that come under the age group 61-90 is 14 


\section{Type of Therapy}

\section{Interpretation}

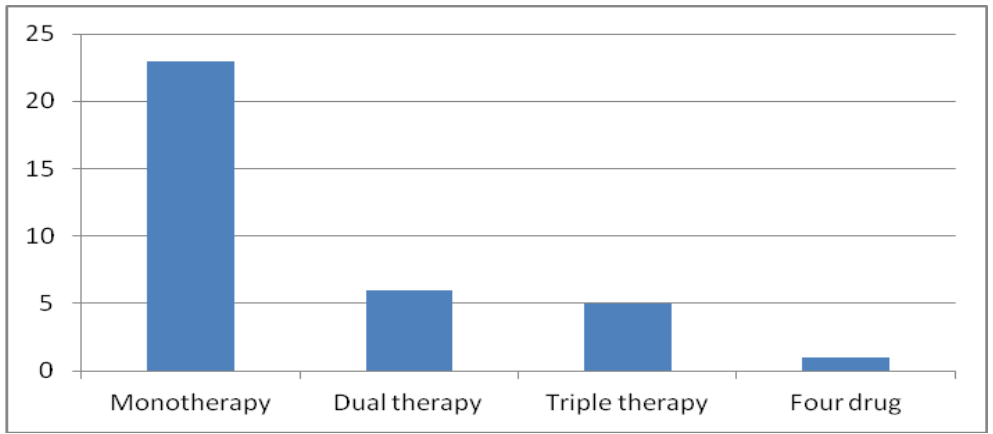

- Mono therapy is prescribed in 23 patients.

- Dual therapy is prescribed in 6 patients.

- Triple therapy is prescribed in 5 patients.

- Four drugs are prescribed in 1 patient.

\section{Drugs}

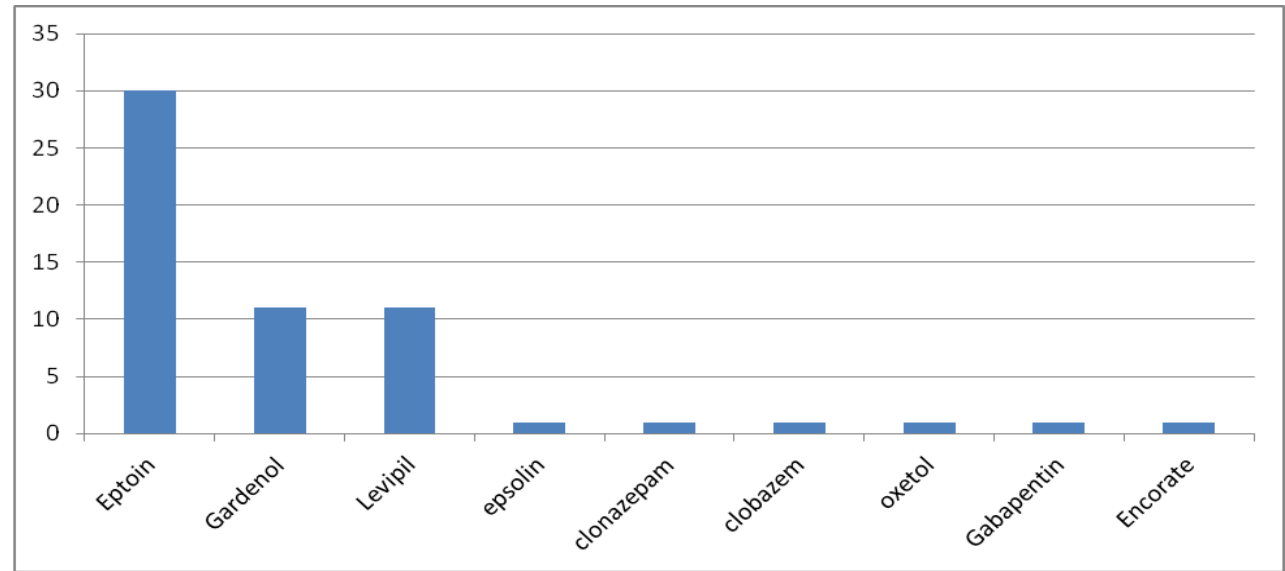

\section{Interpretation}

Most commonly prescribed drug concluded is phenytoin which accounts for $30(85.30 \%)$ in both the genders, followed by phenobarbitone $11(31.18 \%)$ and levitracetam usage was $11(31.18 \%)$.

\section{Dosage Form of Eptoin}

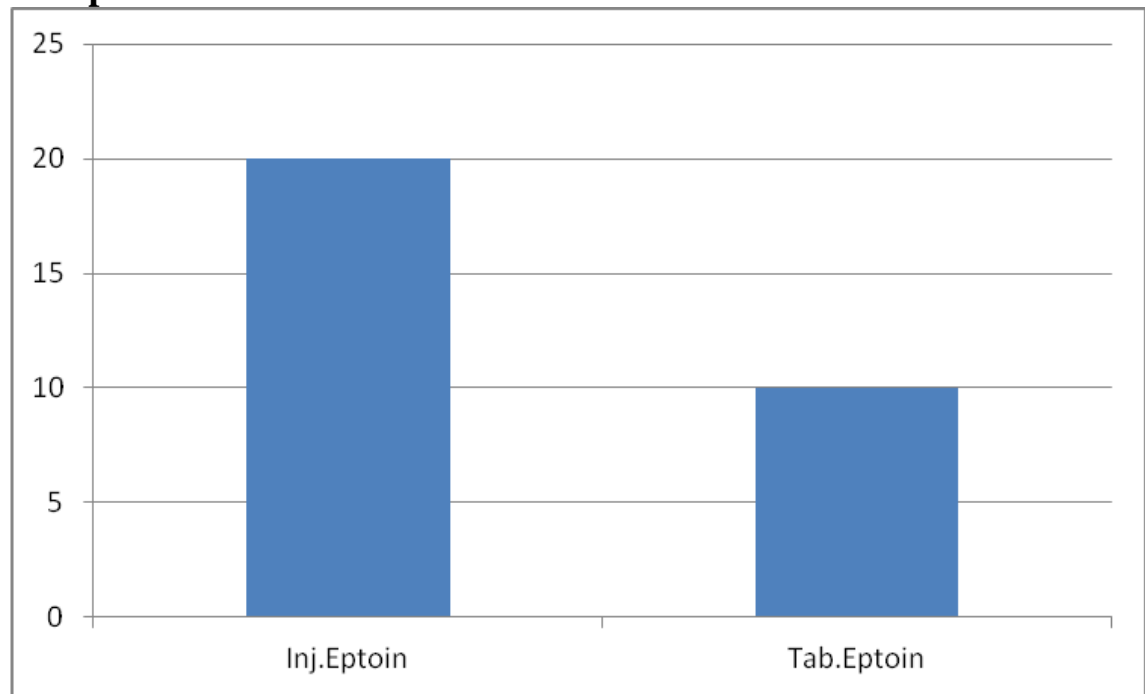

\section{Interpretation}

- Inj.Eptoin are given in 20 patients

- Tab.Eptoin are given in 10 patients 


\section{Dosage Form of Gardenal}

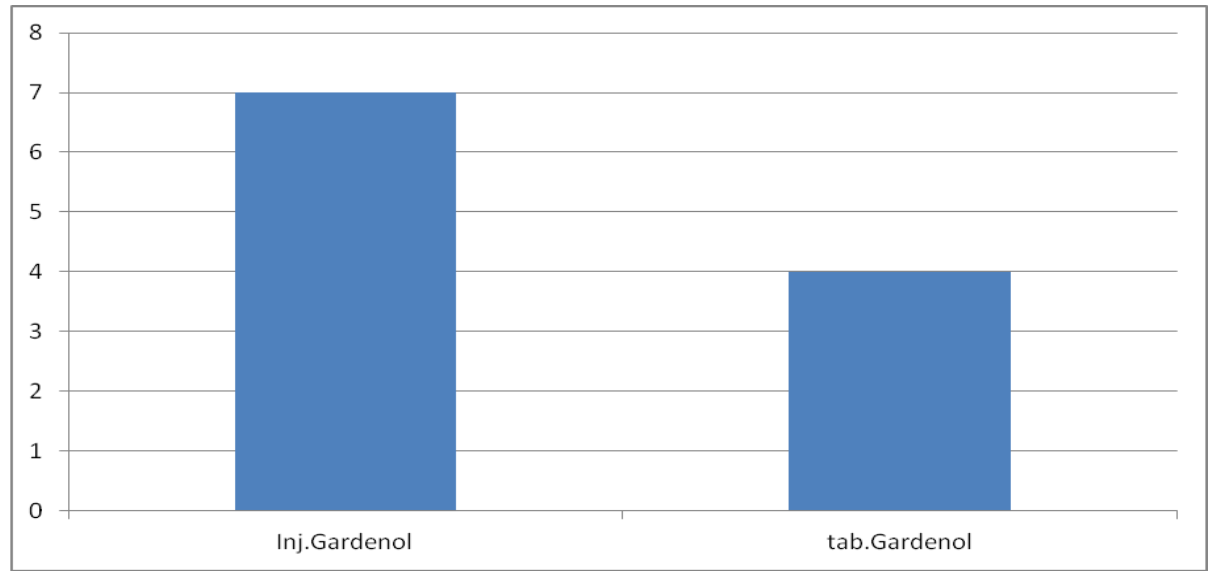

\section{Interpretation}

- Inj.Gardenal is given in 7 patients

- Tab.Gardenol is given in 4 patient

\section{Dosage Form of Levipil}

\section{Interpretation}

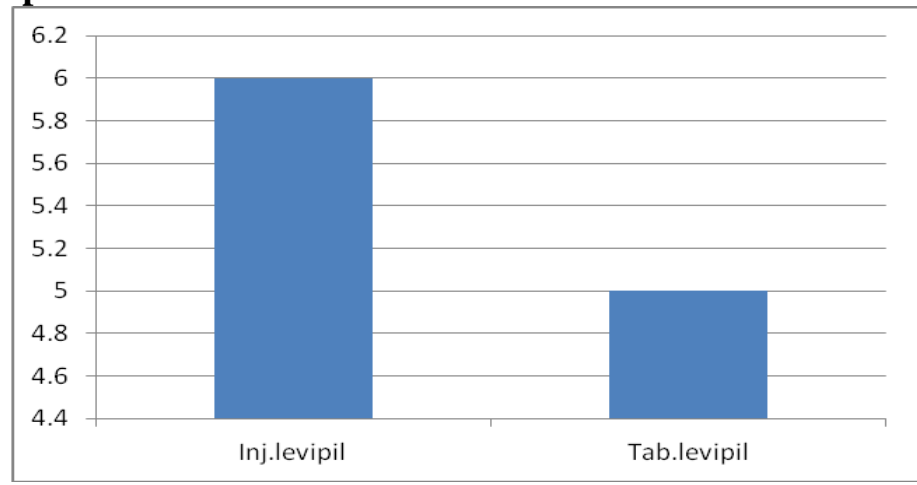

- Inj.levipil is given in 6 patients

- Tab.levipil is given in 5 patients

\section{Drug Therapies}

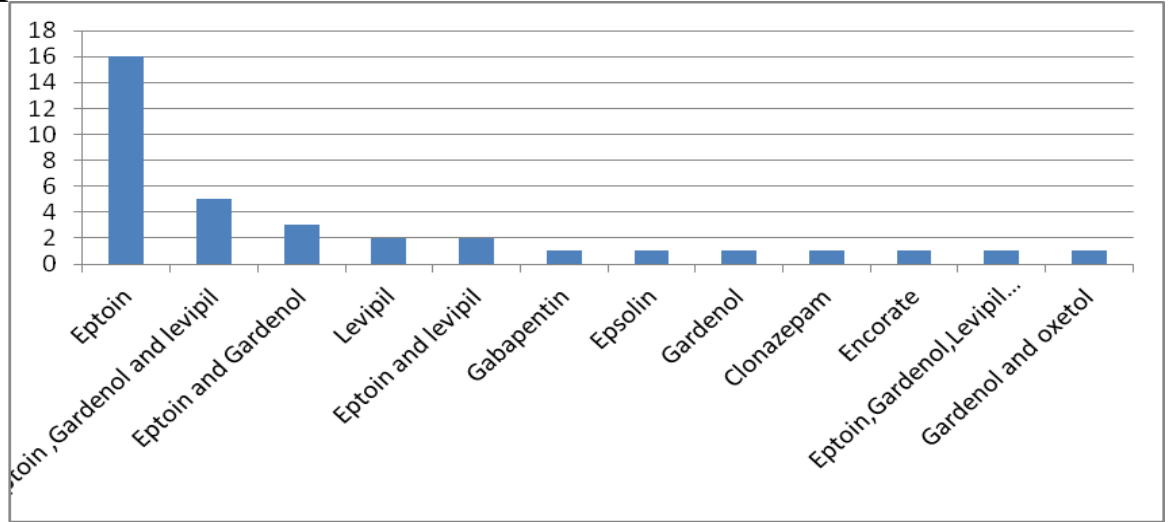

\section{Interpretation}

- In Mono Therapy, Eptoin has been in most cases.

- In Dual Therapy, Eptoin \& Gardenal has been given in most cases.

- In Triple Therapy, Eptoin, Gardenal \& Levipil has been given in most cases.

- The 4 drug combination has been given to only 1 patient and the drugs are Eptoin, Gardenal, Levipil, and Clobazam. 


\section{Discussion}

- The beneficial effects of second generation drugs can also be studied conduction Drug utilization studies in this area.

- A total of 35 epileptic patients were included.

- The incidence of epilepsy was found to be higher in female than in males and also the incidence of epilepsy increases with an increase in age.

- Another finding observed in the study was that Phenytoin was the most frequently prescribed drug followed by Phenobarbital and levitracaetem.

- The above studies justified the use of Phenytoin because it was the equally effective as other AEDs when used in monotherapy, very less incidences of adverse drug reactions (ADR) and also the cost was least

\section{Conclusion}

- Monotherapy is the type of therapy most frequently used in all types of seizures.

- The selection of AEDs is based on efficacy for specific seizure types and epileptic syndromes.

- Most commonly prescribed drug concluded by our study is Phenytoin followed by Phenobarbitone and Levitracaetem usage.

- The percentage of female suffering from epilepsy was higher than males.

- The study shows that most of the patients were from the age group of 60-70 years.

\section{Reference}

[1]. Dhillon S, Sander JW. Epilepsy. In: Walker R, Edwards C, editor. Clinical Pharmacy and Therapeutics. 3 ed. Scotland: Churchill living stone; 2003; 465-466.

[2]. Helms, Quan, Herfindal, Gourley. Textbook of therapeutics drug and disease management 8th ed., pg. nos. 1609, 1611.

[3]. Sridharan R., Epidemiology of Epilepsy, Current Sciences, Vol. 82, No. 6, 2002 March 25th, pg. nos. 664-670.

[4]. Arulkumaran K.S.G. et al., A study on the drug use evaluation of ADEs at multispecialty Tertiary Care Teaching Hospital. International Journal of Pharm. Tech. Research Coden (USA), Vol. 1 (4), 2009 Oct-Dec, pg. nos. 1541-1547.

[5]. Sato, S., Clin. Neurol. (Tokyo), 1964, Vol. 4, pg. nos. 313-324.

[6]. Juul-Jensen. P. and Foldspang, A., Epilepsia, 1983, Vol. 24, pg. nos. 297-312.

[7]. Granieri, E., Rosati, G., Tola, R., Pavoni, M., Paolino, E., Pinna, L. and Monetti, V. C. ibid, 1985, Vol. 24, pg. nos. 502-514.

[8]. Mani K. S. Neurosci. Today, 1997, Vol. 1, pg. nos. 167-174.

[9]. Guberman, A. H. and Bruni, J., Essentials of Clinical Epilepsy, Butterworth Heinemann, Boston, 1999, 2nd ed., pg. nos. 3-10.

[10]. Cockerell, O. C. and Shorvon, S. D., Epilepsy Currents Concepts, Current Medical Literature Ltd., London, 1996, pg. nos. 1-13.

[11]. Placencia, M., Shorvon, S. D., Paredes, V., Bimos, C., Sander, J. W., Suarez, J. and Cascante, S. M., Brain, Vol. 115 1992, pg. nos. 771-782.

[12]. Hauser, W. A., Annegers, J. F. and Kurland, L. T., Epilepsia, 1993, Vol. 34 pg. nos. 453-468.

[13]. Shobhana Mathur, Sumana Sen et al., Asian Journal Utilization Pattern of AEDs and their adverse effects, in a Teaching Hospital, Vol. 3(1), 2010 January-March, pg. nos. 55-59.

[14]. G. Parthasarathi, Karin Nyfort-Hansen, Milap C. Nahata, A textbook of Clinical Pharmacy Practice, 1st ed., pg. no. 362.

[15]. Shih-Hui, Eng-King and Christopher, Pattern of AEDs usage in a tertiary referral hospital in Singapore, Department of Neurology, Singapore, Neurol. Journal. Southeast Asia 1997, pg. nos. 77-85.

[16]. Kasper, Braunwald, Fauci et al., Harrison's principles of Internal Medicine, 16th ed., vol. II, pg. 2357.

[17]. Neligan A., The incidence and prevalence of epilepsy, National general practice study of epilepsy, 2009, Vol II, pg.2.

[18]. Radhakrishnan K, Nayak SD, Kumar SP, Sarma PS. Profile of antiepileptic pharmacotherapy in a tertiary referral center in South India: a pharmacoepidemiologic and pharmacoeconomic study. Epilepsia, 1999; vol. 40, pg. no.179-185. 